\title{
Measurement of the excitation dependence of the Lorentz local-field shift
}

\author{
H. van Kampen, V. A. Sautenkov,* C. J. C. Smeets, E. R. Eliel, and J. P. Woerdman \\ Huygens Laboratory, Leiden University, P.O. Box 9504, 2300 RA Leiden, The Netherlands
}

(Received 3 September 1998)

\begin{abstract}
We have experimentally investigated the resonant optical response of a partially excited high-density atomic potassium vapor under conditions where local-field effects strongly influence the response. We have measured the excitation dependence of the Lorentz local-field shift, and found it to be in excellent agreement with theoretical predictions. [S1050-2947(99)09601-8]
\end{abstract}

PACS number(s): 32.70.Jz, 42.65.An

For dense matter the connection between microscopic electromagnetic properties like atomic and molecular polarizabilities, and a macroscopic response function, such as a susceptibility, is highly nontrivial because of many-body aspects. A widely used procedure to make this connection is to introduce the concept of a local field $E_{\text {loc }}$, as put forward by Lorentz [1]. This field, which is defined at the position of a specific particle, is due to both the externally applied electromagnetic field $E$ and the polarization $P$ of all the other particles. The relation between $E_{\text {loc }}$ and $E$ is given by $[1,2]$

$$
E_{\mathrm{loc}}=E+(4 \pi / 3) P
$$

assuming the virtual cavity to be spherical. Although the local-field concept has been known for more than a century, the interest in the subject is still very much alive [3].

If the material responds linearly to the driving field, one can write $P=N \alpha E_{\text {loc }}$ for the polarization, where $N$ represents the number density of particles and $\alpha$ their polarizability. With the standard relation for the dielectric coefficient $\epsilon=1+4 \pi \chi$, with $\chi=P / E$ the macroscopic linear susceptibility, we obtain the Lorentz-Lorenz relation [4]

$$
\epsilon=1+4 \pi N \alpha\left(\frac{\epsilon+2}{3}\right)
$$

This form explicitly shows that the dense medium has an enhanced dielectric response as compared to a rarefied medium for which $\epsilon=1+4 \pi N \alpha$. The enhancement factor $L$ $=(\epsilon+2) / 3$ is frequently called the local-field correction factor [5]. This factor plays an important role in nonlinear optics of dense media, where effects associated with the $n$ th-order nonlinear susceptibility become enhanced by a factor $L^{n+1}$.

The implications of the local-field ansatz [Eq. (1)] for the resonant electromagnetic response of a system, in particular for a collection of dipole oscillators, were already discussed by Lorentz himself [4]. He showed that the resonance frequency of the dielectric coefficient $\epsilon(\omega)$ of the ensemble is shifted relative to that of the individual oscillator. This shift has become known as the Lorentz local-field shift. Experimental evidence for this shift came almost a century after Lorentz' proposal, in an experiment on the linear and non-

\footnotetext{
*Present address: Physics Department, Texas A\&M University, College Station, TX 77843-4242.
}

linear optical response of a dense atomic vapor in the vicinity of its fundamental atomic resonance frequency [6].

In addition, it has been predicted that the local-field shift of a dense atomic vapor should depend on its degree of excitation [7]. An indirect experimental indication of the existence of an exitation-dependent line shift has recently been given [8]. This prediction has strongly stimulated the study of a whole array of new nonlinear phenomena in dense atomic vapors such as mirrorless optical bistability [9], ultrafast switching effects [10], self-induced transparency [11], piezophotonic switching [12] and lasing without inversion [12]. In the present paper, we report the results of an experimental study of the excitation dependence of the Lorentz local-field shift in a dense atomic vapor.

The usual procedure to introduce the Lorentz local-field shift in the optical response of a dense atomic medium is first to evaluate the atomic polarizability $\alpha(\omega)$ for a dilute vapor in the vicinity of the resonance frequency $\omega_{0}$. Then applying the Lorentz-Lorenz relation [Eq. (2)] yields a dielectric coefficient $\epsilon$ whose real and imaginary parts are shifted to lower frequencies, as compared to $\omega_{0}$, by an amount $\Delta \omega_{L}^{0}$, the Lorentz local-field shift in the zero-excitation approximation.

Using a quantum-electrodynamical calculation that does not make the local-field approximation, Friedberg, Hartmann, and Manassah [7] showed that the Lorentz shift is proportional to the fractional population difference between the ground and excited states $\eta=\left(N_{g}-g_{g} / g_{e} N_{e}\right) / N ; \eta=1$ corresponds to zero excitation and $\eta=0$ to maximum excitation. Here $g_{g}$ and $g_{e}$ are the degeneracies of the ground and excited states, respectively, and $N_{g}$ and $N_{e}$ the corresponding densities. For the dielectric coefficient the authors of Ref. [7] found

$$
\epsilon(\omega)=1+\frac{k N \eta}{\omega-\omega_{0}+\Delta \omega_{L}+\Delta \omega_{\mathrm{NL}}-i \frac{1}{2} \Gamma_{\mathrm{self}}},
$$

with $\Delta \omega_{L}=\eta \Delta \omega_{L}^{0}$ the excitation-dependent Lorentz shift and $\Delta \omega_{L}^{0}=k N / 3$. The constant $k$ is given by $k=f c r_{e} \lambda$, where $r_{e}$ is the classical radius of the electron, $f$ the oscillator strength of the transition, and $\lambda$ the transition wavelength. In Eq. (3) $\Delta \omega_{\mathrm{NL}}$ represents a non-Lorentz contribution to the line shift that does not appear in the theory of Ref. [7] but was found experimentally [6]. Its physical interpretation is subject to controversy $[6,13]$. Our study of the excitation 


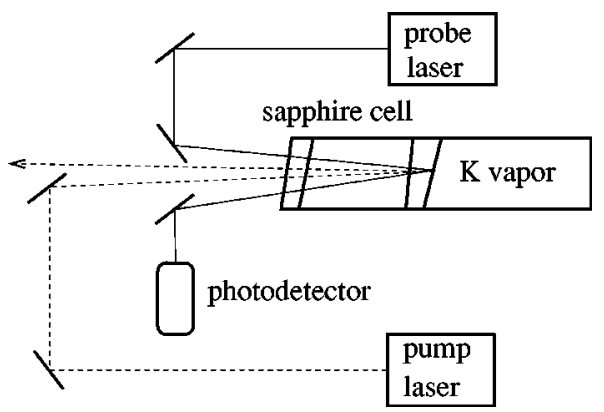

FIG. 1. Experimental pump-probe setup to measure selectivereflection spectra on the fundamental resonance transitions in a dense potassium vapor.

dependence of the line shift provides insight into this matter. $\Gamma_{\text {self }}$ represents the self-broadened width of the line (full width at half maximum). For a dense atomic vapor $(N$ $\left.\approx 10^{17} \mathrm{~cm}^{-3}\right), \Gamma_{\text {self }}$ is proportional to the ground-state density, $\Gamma_{\text {self }}=k \sqrt{g_{g} / g_{e}} N_{g}$. This shows that the self-broadened linewidth is excitation dependent [8].

In our experiment we probe a dense atomic potassium vapor in reflection. In a pump-probe experimental setup (see Fig. 1) we determine the reflectivity of the dielectric-vapor interface on the $D_{1}$ or $D_{2}$ resonance transitions when the vapor is appreciably excited by the beam of a Ti:sapphire pump laser, focussed to a spot size with $50-\mu \mathrm{m}$ diameter (powers up to $500 \mathrm{~mW}$ ). The pump laser is tuned away from the atomic resonance to avoid coherent effects and effects resulting from large inhomogeneities in the spatial distribution of excited-state atoms [14]. The probe laser is an external-cavity semiconductor laser which is scanned over the full spectral line around the resonance transitions, and its power $(0.1 \mathrm{~mW})$ and spot size diameter $(50 \mu \mathrm{m})$ are such that saturation effects are negligible [6]. The vapor cell is completely made of sapphire, which allows us to reach high vapor densities: $N \approx 10^{17} \mathrm{~cm}^{-3}$. At this density the selfbroadened linewidth is so large $\left(\Gamma_{\text {self }} \approx 10 \mathrm{GHz}\right)$ that hyperfine splitttings and the Doppler effect can be ignored.

Typical experimental results are shown in Fig. 2 for a density $N=2.3 \times 10^{17} \mathrm{~cm}^{-3}$. Curve $(a)$ shows the experimental reflectivity spectrum $R(\omega)$ for an unexcited vapor, i.e., with the pump laser switched off. Curve $(b)$ depicts $R(\omega)$ when the vapor is excited by a $400-\mathrm{mW}$ laser beam. The zero on the frequency axis refers to the center frequency $\omega_{0}$ of the absorption spectrum of a low-density $(N$ $\approx 10^{12} \mathrm{~cm}^{-3}$ ) potassium vapor. To improve the signal-tonoise ratios we measured frequency-modulated (FM) reflectivity spectra that are shown in curves $(c)$ and $(d)$, for the unexcited and excited cases, respectively In this case the probe laser is frequency modulated with a modulation depth of $100 \mathrm{MHz}$ at a frequency of $400 \mathrm{~Hz}$. Direct inspection of the FM spectra already yields an important result. With the line center defined as the average of the two frequencies where the FM signal equals zero (see Fig. 2), one observes that the position of the line center differs for curves $(c)$ and $(d)$ : when the vapor becomes partially excited, the center of the spectral line shifts a few $\mathrm{GHz}$ to the blue. Note that, except for a small fraction, this shift is not a light shift or dynamical Stark shift, since its values for both positive and negative detunings of the pump laser are almost the same as

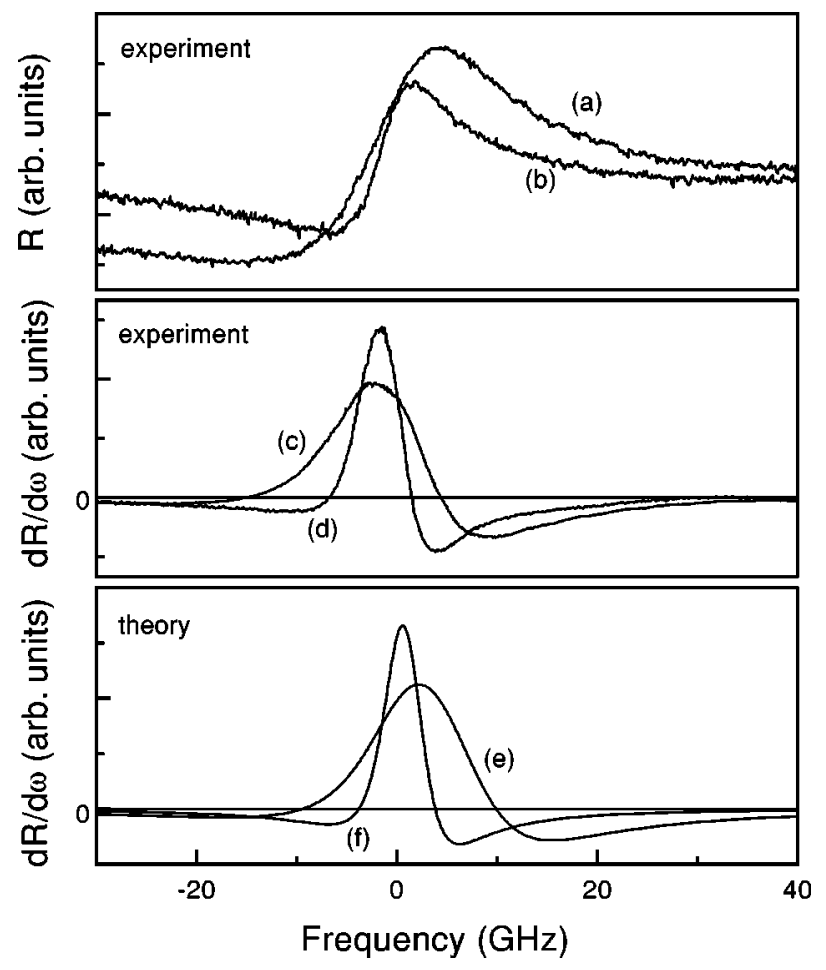

FIG. 2. Experimental reflectivity spectra for a dense potassium vapor $\left(N=2.3 \times 10^{17} \mathrm{~cm}^{-3}\right)$ on the $D_{1}$ transition. Curves $(a)$ and (b) show the reflectivity of the unexcited and excited vapor, respectively. Curve $(c)$ is the frequency-modulated reflectivity in the unexcited case, and curve $(d)$ depicts the same for the excited case. The theoretically obtained frequency-modulated reflectivity spectra for the unexcited and excited vapor are depicted in curves $(e)$ and $(f)$, respectively. In all cases the pump laser is tuned $50 \mathrm{GHz}$ at the low-frequency side of the atomic resonance and produces $400 \mathrm{~mW}$ of output power.

will be shown below. One also sees immediately that the experimental linewidth $\Gamma_{\text {expt }}$, defined as the frequency difference between the two zero crossings (see Fig. 2), is 2.5 times smaller for the partially excited vapor as compared to the unexcited vapor, in agreement with earlier results [8].

Curves $(c)$ and $(d)$ of Fig. 2 are both very well described by the simple expression for $\epsilon(\omega)$ [Eq. (3)]. This is shown from their excellent agreement with the calculated spectra $(e)$ and $(f)$, using the Fresnel formula for the interface reflectivity with appropriate values for the spectral width and population difference $\eta N$. This proves that the spectrum does not become distorted as a result of the partial excitation of the vapor (apart from spectral shifts). It is then allowed [6] to relate the spectral shift and width to the zero crossings of the FM spectra as introduced above [15].

Figure 3 shows the observed shift $\Delta \omega_{\text {expt }}$ as a function of the pump power $P_{\text {pump }}$ for $N=2.3 \times 10^{17} \mathrm{~cm}^{-3}$ ( $D_{1}$ transition). The circles and squares represent our experimental results for pump detunings of -50 and $+50 \mathrm{GHz}$ relative to $\omega_{0}$, respectively. The shift $\Delta \omega_{\text {expt }}$ is clearly power dependent and negative (i.e., the spectral line is shifted to the lowfrequency side of $\omega_{0}$ ). With increasing pump power the shift becomes less negative implying that the line shifts back toward resonance (relative blueshift). We ascribe the difference in shift for positive and negative pump detuning to the light shift (an estimate of the light shift gives the proper 


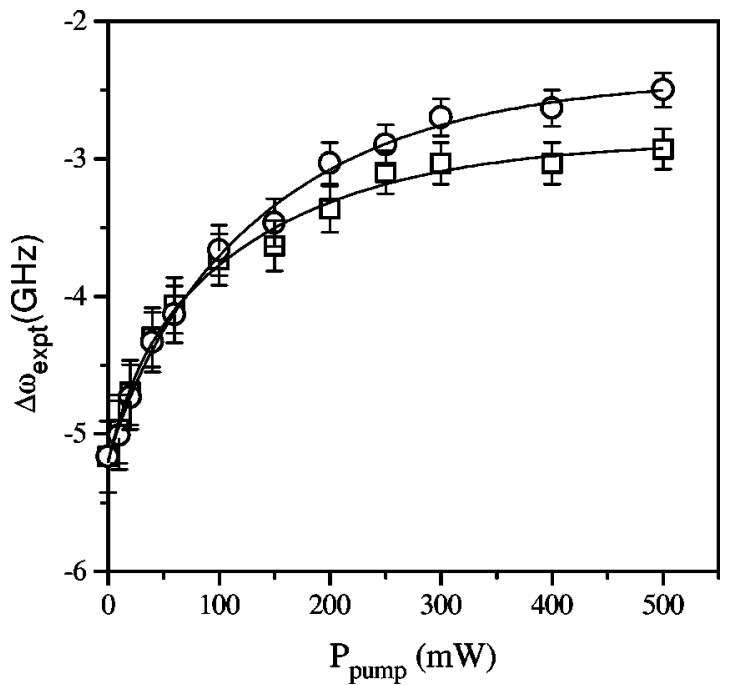

FIG. 3. Experimental values of the shift $\Delta \omega_{\text {expt }}$ of the $D_{1}$ line as a function of the pump laser power for a dense potassium vapor $\left(N=2.3 \times 10^{17} \mathrm{~cm}^{-3}\right)$. The detuning of the pump laser from resonance equals $-50 \mathrm{GHz}$ (circles) and $+50 \mathrm{GHz}$ (squares) with respect to $\omega_{0}$, the center of the low-density absorption spectrum. The solid curves through the data have been drawn to guide the eye.

order of magnitude). This difference is small as compared to the total shift $\Delta \omega_{\text {expt }}$ (see Fig. 3), and will therefore be neglected.

Because the saturation behavior of a dense alkali vapor is highly nontrivial, it is hard to determine the fractional population difference $\eta$ acurrately from the values of the pump power. Rather we determine $\eta$, for each value of the pump power, from a direct comparison between an experimental spectrum and a calculated spectrum while ignoring any spectral shift. To calculate the spectrum we use, as discussed above, the Fresnel formula for the reflectivity and the dielectric function of Eq. (3); in the latter we use the experimental value for the linewidth $\Gamma_{\text {expt }}$, deduced from Fig. 2. The comparison directly yields $\eta$. To check the internal consistency of our method to determine $\eta$, in Fig. 4 we plot the resulting values of $\eta$ versus the experimental linewidth for $N=$ $2.3 \times 10^{17} \mathrm{~cm}^{-3}$ ( $D_{1}$ line) because the width is expected to depend linearly on $\eta$ [8]. Note that, since $\eta$ is the fractional population difference, the width $\Gamma_{\text {expt }}$ has been normalized to its value in the limit of zero excitation $\Gamma_{\text {expt }}^{0}$. The dashed line in Fig. 4 shows the expected result based on a quasistatic picture of the dipole-dipole collisions; such a picture applies at this density [8]. The excellent agreement between this model and our experimental data, obtained without any fit parameter, increases our confidence that our method to determine $\eta$ is reliable. Note that the atomic ground state becomes strongly depleted in the present experiment: at the highest pump power that was used the fractional population difference reaches a value $\eta=0.23$. In this case the fraction of atoms that resides in the ground state is less than half $\left(N_{g} / N \approx 0.42\right)$. Radiation trapping plays an important role in achieving such a strong depletion of the ground state [16]. The excited-state atoms are statistically distributed over the two fine-structure levels of the excited $4 p$ state.

We will now discuss our key result, namely, the observed excitation dependence of the spectral shift $\Delta \omega_{\text {expt }}$. To do so

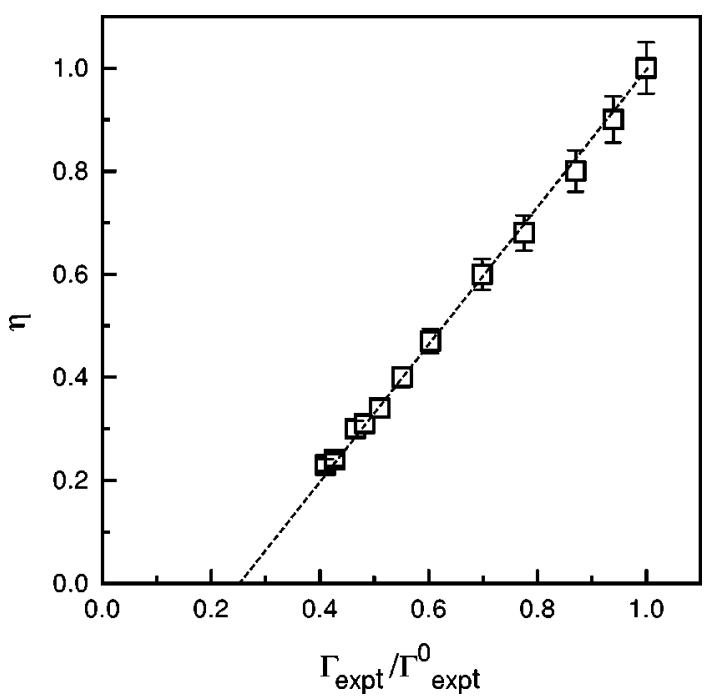

FIG. 4. The population difference $\eta$ as a function of the normalized width $\Gamma_{\text {expt }} / \Gamma_{\text {expt }}^{0}$ of the selective-reflection spectrum for the potassium $D_{1}$ line at a density $N=2.3 \times 10^{17} \mathrm{~cm}^{-3}$. The dashed line corresponds to theory, as discussed in the text.

we normalize the shift to its value for zero pump power $\Delta \omega_{\text {expt }}^{0}$. Figure 5 shows the normalized shift $\Delta \omega_{\text {expt }} / \Delta \omega_{\text {expt }}^{0}$ as a function of the experimentally obtained values of the fractional population difference $\eta$ for the $D_{1}$ - and $D_{2}$ - transitions, respectively $\left(N=2.3 \times 10^{17} \mathrm{~cm}^{-3}\right)$. The dashed lines are the results of a linear fit through the data points: $\Delta \omega_{\text {expt }} / \Delta \omega_{\text {expt }}^{0}=\alpha \eta+(1-\alpha)$. Our results are clearly well described by this function, and we find that $\alpha=0.62$ for the $D_{1}$ line whereas, $\alpha=0.82$ for the $D_{2}$ line. This implies that $\Delta \omega_{\text {expt }}$ contains both an excitation-dependent part, which we identify with the Lorentz shift, and an excitation-independent part. Our results imply that the Lorentz shift is linear in the degree of excitation, $\Delta \omega_{L}=\eta \Delta \omega_{L}^{0}$, in perfect agreement with theoretical predictions [7]. Our experimental values for

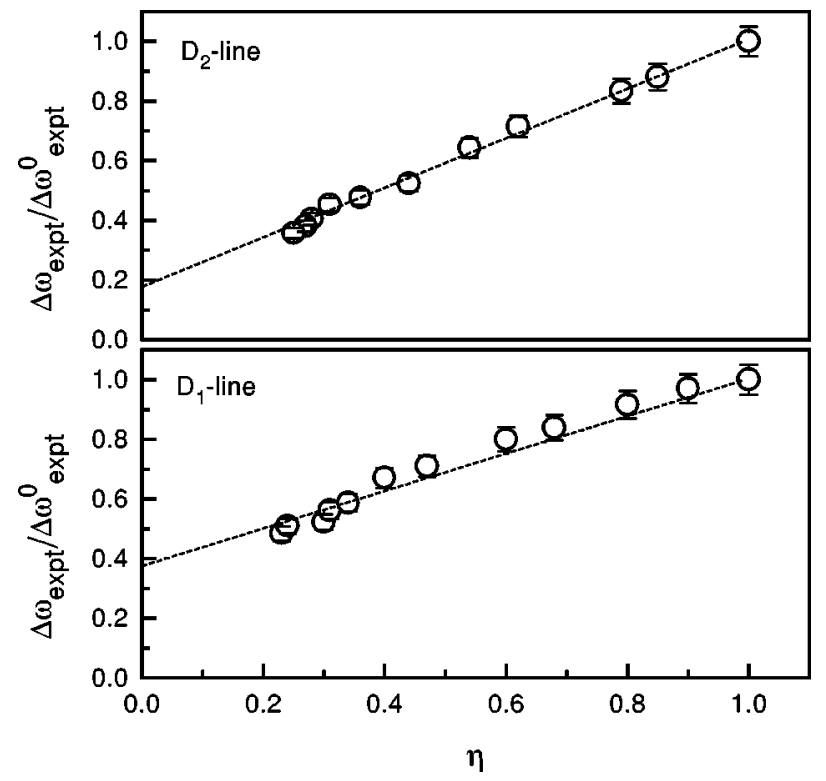

FIG. 5. The normalized shift $\Delta \omega_{\text {expt }} / \Delta \omega_{\text {expt }}^{0}$ as a function of the fractional population difference $\eta$ for the potassium $D_{1}$ and $D_{2}$ lines $\left(N=2.3 \times 10^{17} \mathrm{~cm}^{-3}\right)$. The dashed lines represent linear fits. 
$\alpha$ are in excellent agreement with the ratios $\Delta \omega_{L}^{0} / \Delta \omega_{\text {expt }}$ for the potassium $D_{1}$ and $D_{2}$ transitions as found by Maki et al. [6] in an experiment at $\eta=1$; this confirms our identification of the excitation-dependent part of the normalized shift with the Lorentz shift.

Following the same argument, the excitation-independent part of $\Delta \omega_{\text {expt }}$ is identified with the non-Lorentz shift $\Delta \omega_{\mathrm{NL}}$ which was previously observed in the zero-excitation limit and attributed to van der Waals interactions between groundstate atoms [6]. Recently this interpretation was rejected, and an alternative interpretation put forward. This involves the interaction between the atoms and the wall and the short penetration length of the incident laser field at high atomic densities [13]. In view of our result that $\Delta \omega_{\mathrm{NL}}$ is essentially excitation independent, the latter interpretation is deemed to be more plausible.

In conclusion, we have measured the reflectivity of a partially excited atomic potassium vapor for densities where local-field effects are important. We have experimentally shown that the Lorentz local-field shift depends linearly on the degree of excitation, in perfect agreement with theoretical predictions [7]. We have also confirmed the existence of a non-Lorentz (excitation-independent) contribution to the spectral shift, and made plausible that this is not a collisional shift. The present experiment underlines that the local-field ansatz of Lorentz provides an excellent quantitative description of the optical response of a high-density atomic vapor around resonance. It provides solid footing for theoretical predictions of exciting phenomena in nonlinear optics based on the excitation dependence of the Lorentz shift. Apart from mirrorless optical bistability [9], experimental confirmation of these nonlinear phenomena [10-12] has not yet been reported.

This work is part of the research program of the "Stichting voor Fundamenteel Onderzoek der Materie,' and was made possible by financial support from the "Nederlandse Organisatie voor Wetenschappelijk Onderzoek."
[1] H.A. Lorentz, Verh. Kon. Ned. Acad. Wet. 18, 1 (1879).

[2] D.E. Aspnes, Am. J. Phys. 50, 704 (1982).

[3] P. de Vries and A. Lagendijk, Phys. Rev. Lett. 81, 1381 (1998); F.J.P Schuurmans, D.T.N. de Lang, G.H. Wegdam, R. Sprik, and A. Lagendijk, ibid. 80, 5077 (1998); A. Lagendijk, B. Nienhuis, B.A. van Tiggelen, and P. de Vries, ibid. 79, 657 (1997); L. Jönsson, Z.H. Levine, and J.W. Wilkins, ibid., 76, 1372 (1996); G.L.J.A. Rikken and Y.A.R.R. Kessener, ibid., 74, 880 (1995).

[4] H.A. Lorentz, The Theory of Electrons, 2nd ed. (Dover, New York, 1952), Secs. 117-136 and Note 54.

[5] N. Bloembergen, Nonlinear Optics (Benjamin, New York, 1965).

[6] J.J. Maki, M.S. Malcuit, J.E. Sipe, and R.W. Boyd, Phys. Rev. Lett. 67, 972 (1991); J.J. Maki, W.V. Davis, R.W. Boyd, and J.E. Sipe, Phys. Rev. A 46, 7155 (1992).

[7] R. Friedberg, S.R. Hartmann, and J.T. Manassah, Phys. Rep. 7, 101 (1973); Phys. Rev. A 42, 494 (1990); J.T. Manassah, Phys. Rep. 101, 359 (1983).

[8] V.A. Sautenkov, H. van Kampen, E.R. Eliel, and J.P. Woerd- man, Phys. Rev. Lett. 77, 3327 (1996).

[9] M.P. Hehlen, H.U. Güdel, Q. Shu, J. Rai, S. Rai, and S.C. Rand, Phys. Rev. Lett. 73, 1103 (1994); R. Friedberg, S.R. Hartmann, and J.T. Manassah, Phys. Rev. A 39, 3444 (1989).

[10] M.E. Crenshaw, M. Scalora, and C.M. Bowden, Phys. Rev. Lett. 68, 911 (1992).

[11] C.R. Stroud and C.M. Bowden, Opt. Commun. 67, 387 (1988).

[12] A.S. Manka, J.P. Dowling, C.M. Bowden, and M. Fleischhauer, Quantum Opt. 6, 371 (1994).

[13] J. Guo, J. Cooper, and A. Gallagher, Phys. Rev. A 53, 1130 (1996); P. Wang, A. Gallagher, and J. Cooper, ibid. 56, 1598 (1997).

[14] H. van Kampen, V.A. Sautenkov, A.M. Shalagin, E.R. Eliel, and J.P. Woerdman, Phys. Rev. A 56, 3569 (1997).

[15] The shift $\Delta \omega_{\text {expt }}$ is not exactly equal to the shift in $\epsilon(\omega)$ because of the nonlinearity of the relation between the reflectivity $R(\omega)$ and the dielectric coefficient $\epsilon(\omega)$. This difference is about $2 \%$, which is small compared to our experimental accuracy.

[16] T. Holstein, Phys. Rev. 83, 1159 (1951). 